\title{
Heat transfer between nanoparticles: Thermal conductance for near-field interactions
}

\author{
A. Pérez-Madrid* and J. M. Rubí ${ }^{\dagger}$ \\ Departament de Física Fonamental, Facultat de Física, Universitat de Barcelona, Avenida Diagonal 647, 08028 Barcelona, Spain \\ L. C. Lapas \\ Instituto de Física and Centro Internacional de Física da Matéria Condensada, Universidade de Brasília, Caixa Postal 04513, \\ 70919-970 Brasília, Distrito Federal, Brazil
}

(Received 11 January 2008; published 11 April 2008)

\begin{abstract}
We analyze the heat transfer between two nanoparticles separated by a distance lying in the near-field domain in which energy interchange is due to the Coulomb interactions. The thermal conductance is computed by assuming that the particles have charge distributions characterized by fluctuating multipole moments in equilibrium with heat baths at two different temperatures. This quantity follows from the fluctuation-dissipation theorem for the fluctuations of the multipolar moments. We compare the behavior of the conductance as a function of the distance between the particles with the result obtained by means of molecular dynamics simulations. The formalism proposed enables us to provide a comprehensive explanation of the marked growth of the conductance when decreasing the distance between the nanoparticles.
\end{abstract}

DOI: 10.1103/PhysRevB.77.155417 PACS number(s): 82.60.Qr, 65.80. $+\mathrm{n}$

\section{INTRODUCTION}

The study of energy transfer mechanisms at the nanoscale ${ }^{1}$ has aroused increasing interest due to the emergence of the interdisciplinary field of nanoscience where such wide-ranging fields as, for example, solid state physics, ${ }^{2}$ nanothermodynamics, ${ }^{3-5}$ or electrical engineering ${ }^{6}$ coexist. One of the basic problems in this field is to determine the energy exchange between two nanoparticles (NPs) at different temperatures. The way in which this energy is transferred depends crucially on the distance between the particles. For sufficiently large distances, heat exchange proceeds via thermal radiation, through emission or absorption of photons, whereas at smaller distances, recent molecular dynamics simulations have shown that the Coulomb interaction (nearfield radiation) is the dominant mechanism. ${ }^{7}$

For near-field interactions, the thermal conductance was calculated under the assumption that both NPs behave as effective dipoles at different temperatures. ${ }^{7}$ Hence, since these dipoles undergo thermal fluctuations, the fluctuationdissipation theorem (FDT) $)^{8-11}$ provides the energy which dissipates into heat in each NP. It was found that the heat and therefore the conductance vary according to $d^{-6}$, a very different behavior from the one observed in the case of thermal radiation: $d^{-2}$. The $d^{-6}$ behavior is found in many interactions of the same kind ${ }^{12,13}$ (van der Waals potential, fluorescence resonance energy transfer, and nonradiative coupling between a fluorescent emitter and a metallic nanoparticle). Molecular dynamics simulations agree with the dipole-dipole model when the two NPs are separated by a distance on the order of a few nanometers. However, near contact, the conductance deviates dramatically from the prediction of the dipole model, as the simulations show. This behavior is a consequence of the fact that when particles become very close, the position of the atoms is highly correlated; consequently, the charge distributions become nonsymmetric and cannot be described merely as two interacting dipoles. To account for this distortion of the distribution of charges, a more general formalism which focuses more convoluted interactions in- volving higher order multipoles aside from the dipoles is required.

Our purpose in this paper is to provide this general formalism enabling us to analyze the behavior of the conductance beyond the dipolar approximation. We will use the linear response theory to derive an expression of the FDT for the fluctuations of the higher order multipoles. In particular, we will focus on the quadrupolar contributions to the conductance, which are able to reproduce the behavior observed in the simulations for some sizes of the NPs.

The paper is organized as follows. In Sec. II, we present the multipolar expansion of the Coulomb forces ${ }^{14}$ between both NPs and derive a general expression of the FDT valid for multipoles of any order which leads to the heat transfer between the NPs. In Sec. III, we analyze the particular case of quadrupolar contributions and derive the expresion of the conductance. We compare our result with the molecular dynamics simulations. ${ }^{7}$ Finally, in Sec. IV. We emphasize our main conclusions.

\section{HEAT TRANSFER BETWEEN TWO NANOPARTICLES}

In this section, we will study the near-field radiative heat transfer flux between two NPs which interact through the Coulomb forces.

\section{A. Multipolar expansion}

To analyze the Coulomb interaction between two NPs (see Fig. 1), it is necessary to know the charge distribution inside each of them. This can be performed by specifying their multipole moments so that the multipole moment of order $n$ of the NPi, $\hat{\mathbf{M}}_{(i)}^{(n)}$, can be defined as ${ }^{15}$

$$
\hat{M}_{(i) ; \alpha}^{(n)}(\mathbf{r})=\frac{1}{n !} \sum_{\mathbf{r}} e_{\mathbf{r}} r^{2 n+1} X_{\alpha}^{(n)}(\mathbf{r}),
$$

where $e_{\mathbf{r}}$ is the charge at the position $\mathbf{r}$ inside the NP and the $X_{\alpha}^{(n)}(\mathbf{r})$ are symmetric irreducible tensors (see Appendix A 


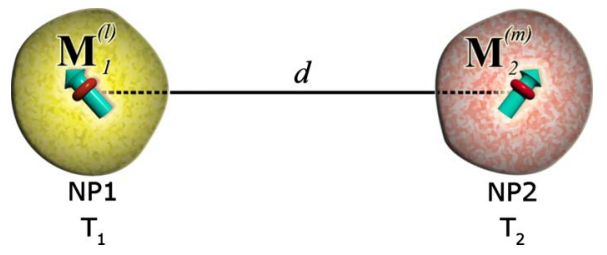

FIG. 1. (Color online) Schematic illustration of the interaction between two NPs (NP1 and NP2) with temperatures $T_{1}$ and $T_{2}$, respectively. Each NP is assimilated to a multipole moment (moments $\mathbf{M}_{1}^{(l)}$ and $\mathbf{M}_{2}^{(m)}$ ) and is separated by a distance $d$ between their centers.

for more details) where $\alpha=\left(\alpha_{1}, \ldots, \alpha_{n}\right)$ and $\alpha_{j}=1,2,3$ for $j=1, \ldots, n$. Thus, the case $n=0$ corresponds to the monopole $\hat{M}_{(i) ; \alpha}^{(0)}(\mathbf{r})=\sum_{\mathbf{r}} e_{\mathbf{r}}, \quad n=1$ is related to the dipole moment $\hat{M}_{(i) ; \alpha}^{(1)}(\mathbf{r})=\Sigma_{\mathbf{r}} e_{\mathbf{r}} r_{\alpha_{1}}$, and $n=2$ for the quadrupole moment $\hat{M}_{(i) ; \alpha}^{(2)}(\mathbf{r})=1 / 2 \Sigma_{\mathbf{r}} e_{\mathbf{r}}\left(3 r_{\alpha_{1} \alpha_{2}}-\delta_{\alpha_{1} \alpha_{2}}\right)$. Hence, in terms of the spherical surface tensors $Y_{\alpha}^{(n)}(\hat{\mathbf{r}})$, given in Eq. (A5), and the unit vector $\hat{\mathbf{r}}$ related to $\mathbf{r}$, Eq. (1) adopts the equivalent form,

$$
\hat{M}_{(i) ; \alpha}^{(n)}(\mathbf{r})=\frac{1}{n !} \sum_{\mathbf{r}} e_{\mathbf{r}} r^{n} Y_{\alpha}^{(n)}(\hat{\mathbf{r}}) .
$$

The above mentioned interaction between these NPs modifies their respective Hamiltonians. The interaction between $\mathrm{NP} i$ with $\mathrm{NP} j$ introduces a time-dependent perturbation $\hat{H}_{(i j)}$ in its Hamiltonian which can be written as a multipolar expansion, ${ }^{14}$

$$
\hat{H}_{(i j)}=\sum_{m=0}^{\infty} c_{m} \hat{\mathbf{M}}_{(i)}^{(m)} \odot \hat{\mathbf{V}}_{(i, j)}^{(m)}(t),
$$

with $c_{m}=1 /(2 m-1) ! !, \odot$ stands for the full contraction of indexes, and $\hat{\mathbf{M}}_{(i)}^{(m)} \odot \hat{\mathbf{V}}_{(i, j)}^{(m)} \equiv \hat{M}_{(i) ; \alpha}^{(m)} \hat{V}_{(i, j) ; \alpha^{*}}^{(m)}$. In addition,

$$
\hat{V}_{(i, j) ; \alpha}^{(m)}=\nabla_{\alpha_{1}} \cdots \nabla_{\alpha_{m}} \hat{\mathbf{V}}_{(i, j)}(d),
$$

with $\hat{\mathbf{V}}_{(i, j)}(d)$ being the interaction potential between both NPs and $d$ the separation between their centers. In terms of the first contributions, the perturbation can be expressed as

$$
\hat{H}_{(i j)}=\hat{\mathbf{M}}_{(i)}^{(0)} \hat{\mathbf{V}}_{(i, j)}+\hat{\mathbf{M}}_{(i)}^{(1)} \cdot \hat{\mathbf{V}}_{(i, j)}^{(1)}+\frac{1}{3} \hat{\mathbf{M}}_{(i)}^{(2)} \odot \hat{\mathbf{V}}_{(i, j)}^{(2)}+\cdots,
$$

where $-\hat{\mathbf{V}}_{(i, j)}^{(1)}$ is the electric field induced in the NPi, $-\hat{\mathbf{V}}_{(i, j)}^{(2)}$ is the gradient of this induced field, and $\hat{\mathbf{M}}_{(i)}^{(2)}$ is the conjugated quadrupolar moment.

Likewise, the electrostatic potential admits a multipolar expansion as well,

$$
\hat{\mathbf{V}}_{(i, j)}(d)=\sum_{n=0}^{\infty} c_{n} \mathbf{G}_{(i, j)}^{(n)} \odot \hat{\mathbf{M}}_{j}^{(n)},
$$

which expresses the fact that the potential acting on the $\mathrm{NP} i$ depends on the charge distribution in the NP $j$. Here, $\hat{\mathbf{M}}_{(j)}^{(n)}$ are the multipolar moments of the $\mathrm{NP} j$ and

$$
\begin{aligned}
G_{(i, j) ; \alpha}^{(n)} & =\frac{(-1)^{n}}{4 \pi \varepsilon_{0}} \nabla_{\alpha_{1}} \cdots \nabla_{\alpha_{n}} \frac{1}{d} \\
& =\frac{1}{4 \pi \varepsilon_{0}} X_{\alpha}^{(n)}(\mathbf{d})=\frac{1}{4 \pi \varepsilon_{0} d^{n+1}} Y_{\alpha}^{(n)}(\hat{\mathbf{d}})
\end{aligned}
$$

is the Green's propagator, with $\mathbf{d}$ the vector connecting the centers of the particles and $\hat{\mathbf{d}}$ the corresponding unit vector. Thus, from Eq. (6),

$$
\hat{\mathbf{V}}_{(i, j)}^{(m)}=\sum_{n=0}^{\infty} c_{n} \mathbf{G}_{(i, j)}^{(m, n)} \odot \hat{\mathbf{M}}_{j}^{(n)},
$$

where $\mathbf{G}_{(i, j)}^{(m, n)}$ is defined through

$$
\mathbf{G}_{(i, j) ; \beta, \alpha}^{(m, n)}=\nabla_{\beta_{1}} \cdots \nabla_{\beta_{m}} G_{(i, j) ; \alpha}^{(n)} .
$$

\section{B. Heat transfer from the fluctuation-dissipation theorem}

In the linear response regime, the multipolar moments can be expressed as

$$
\mathbf{M}_{(i)}^{(n)}(\omega)=\frac{1}{c_{n}} \sum_{m=0}^{\infty} \mathbf{P}_{(i)}^{(n, m)}(\omega) \odot \mathbf{V}_{(i, j)}^{(m)}(\omega),
$$

where $\mathbf{P}^{(n, m)}(\omega)$ are the multipolar polarizabilities which may, in general, depend on frequency.

The energy transferred between the particles and converted into heat can be obtained from the linear response theory. ${ }^{10,11}$ One obtains (see Appendix B)

$$
\begin{aligned}
Q_{i \rightarrow j}= & \frac{-i \omega \varepsilon_{0}}{4} \sum_{n, m=0}^{\infty}\left\{c_{n}\left\langle\mathbf{V}_{(i, j)}^{(n) *} \odot \mathbf{P}_{(j)}^{(n, m)} \odot \mathbf{V}_{(i, j)}^{(m)}\right\rangle\right. \\
& \left.-c_{m}\left\langle\mathbf{V}_{(i, j)}^{(m)} \odot \mathbf{P}_{(j)}^{(m, n) *} \odot \mathbf{V}_{(i, j)}^{(n) *}\right\rangle\right\},
\end{aligned}
$$

where the symbol $*$ stands for the complex conjugated and the brakets express thermal average.

According to Eq. (8), the term in Eq. (11) containing the thermal average can be transformed as

$$
\begin{aligned}
\sum_{n, m=0}^{\infty} & \left\langle\mathbf{V}_{(i, j)}^{(n) *} \odot \mathbf{P}_{(j)}^{(n, m)} \odot \mathbf{V}_{(i, j)}^{(m)}\right\rangle \\
& =\sum_{l, k=0}^{\infty} c_{l} c_{k} \sum_{n, m=0}^{\infty} \times\left\langle\mathbf{M}_{(i)}^{(l) *} \odot \mathbf{G}^{(l, n)} \odot \mathbf{P}_{(j)}^{(n, m)} \odot \mathbf{G}^{(m+k)} \odot \mathbf{M}_{i}^{(k)}\right\rangle \\
& =\sum_{l, k=0}^{\infty} c_{l} c_{k}\left\langle\mathbf{M}_{(i)}^{(l) *} \odot \mathbf{S}_{(j)}^{(l, k)} \odot \mathbf{M}_{i}^{(k)}\right\rangle
\end{aligned}
$$

where we have defined

$$
\mathbf{S}_{(j)}^{(l, k)}=\sum_{n, m=0}^{\infty} \mathbf{G}^{(l, n)} \odot \mathbf{P}_{(j)}^{(n, m)} \odot \mathbf{G}^{(m, k)} .
$$

Moreover, from Eqs. (A3), (7), and (9), one can prove that the $\mathbf{S}_{(j)}^{(l, k)}$ are symmetric tensors. Therefore, making use of Eq. (13), Eq. (11) becomes 


$$
Q_{i \rightarrow j}=\frac{-i \omega \varepsilon_{0}}{4} \sum_{l, k=0}^{\infty} c_{l} c_{k}\left\{\left\langle\mathbf{M}_{(i)}^{(l) *} \odot \mathbf{S}_{(j)}^{(l, k)} \odot \mathbf{M}_{i}^{(r)}\right\rangle-\text { c.c. }\right\} .
$$

The dependence of the energy transferred on the distance $d$ resides in $\mathbf{S}_{(j)}^{(l, k)}$, as follows from Eq. (13) and the expression of the propagators given in Eqs. (7) and (9). The multipolemultipole correlation can be obtained by using the FDT, $8,10,11$

$$
\left\langle\mathbf{M}_{(i)}^{(l) *} \mathbf{M}_{(i)}^{(k)}\right\rangle=\frac{-i \varepsilon_{0}}{\pi \omega c_{l} c_{k}}\left(\mathbf{P}_{(i)}^{(l, k)}-\mathbf{P}_{(i)}^{(k, l) *}\right) \Theta\left(\omega, T_{i}\right),
$$

where $\Theta\left(\omega, T_{i}\right)=\hbar \omega\left\{1 / 2+1 / \exp \left(\hbar \omega / k T_{i}-1\right)\right\}$ is the mean energy of an oscillator. As an illustration, for the dipolar case, ${ }^{7}$ we obtain

$$
\left\langle\mathbf{M}_{(i)}^{(1) *} \mathbf{M}_{(i)}^{(1)}\right\rangle=\frac{-i \varepsilon_{0}}{\pi \omega}\left(\mathbf{P}_{(i)}^{(1,1)}-\mathbf{P}_{(i)}^{(1,1) *}\right) \Theta\left(\omega, T_{i}\right),
$$

where $\mathbf{P}_{(i)}^{(1,1)}$ is the dipole-dipole polarizability which we assume to be given through

$$
P_{(i) ; \alpha, \beta}^{(1,1)}=\alpha_{(i)}(\omega) \Delta_{\alpha, \beta}^{(1)},
$$

and $\triangle_{\alpha, \beta}^{(1)}=\delta_{\alpha_{1} \beta_{1}}$ with $\alpha_{(i)}(\omega)=\alpha_{(i)}(\omega)+i \alpha_{(i)}^{\prime \prime}(\omega)$. Hence, Eq. (16) becomes

$$
\left\langle M_{(i) ; \alpha}^{(1) *} M_{(i) ; \beta}^{(1)}\right\rangle=\frac{2 \varepsilon_{0}}{\pi \omega} \alpha_{(i)}^{\|}(\omega) \Theta\left(\omega, T_{i}\right) \delta_{\alpha_{1} \beta_{1}} .
$$

For the quadrupolar case, one has

$$
\left\langle\mathbf{M}_{(i)}^{(2) *} \mathbf{M}_{(i)}^{(2)}\right\rangle=\frac{-i \varepsilon_{0}}{9 \pi \omega}\left(\mathbf{P}_{(i)}^{(2,2)}-\mathbf{P}_{(i)}^{(2,2) *}\right) \Theta\left(\omega, T_{i}\right),
$$

where $\mathbf{P}_{(i)}^{(2,2)}$ is the quadrupole-quadrupole polarizability given through

$$
P_{(i) ; \alpha, \beta}^{(2,2)}=\beta_{(i)}(\omega) \Delta_{\alpha, \beta}^{(2)},
$$

with $\beta_{(i)}(\omega)=\beta_{(i)}(\omega)+i \beta_{(i)}^{\prime \prime}(\omega)$ and

$$
\Delta_{\alpha, \beta}^{(2)}=\frac{1}{2}\left(\delta_{\alpha_{1} \beta_{1}} \delta_{\alpha_{2} \beta_{2}}+\delta_{\alpha_{1} \beta_{2}} \delta_{\alpha_{2} \beta_{1}}\right) b-\frac{1}{3} \delta_{\alpha_{1} \alpha_{2}} \delta_{\beta_{1} \beta_{2}}
$$

is the isotropic tetradric. Thus, with Eqs. (20) and (21), Eq. (19) is written as

$$
\left\langle M_{(i) ; \alpha}^{(2) *} M_{(i) ; \beta}^{(2)}\right\rangle=\frac{2 \varepsilon_{0}}{\pi \omega\left(c_{2}\right)^{2}} \beta_{(i)}^{\|}(\omega) \Theta\left(\omega, T_{i}\right) \Delta_{\alpha, \beta}^{(2)} .
$$

Up to the quadrupolar order, one has to take into account also the cross correlation dipole quadrupole,

$$
\left\langle\mathbf{M}_{(i)}^{(1) *} \mathbf{M}_{(i)}^{(2)}\right\rangle=\frac{-i \varepsilon_{0}}{\pi \omega c_{2}}\left(\mathbf{P}_{(i)}^{(1,2)}-\mathbf{P}_{(i)}^{(1,2) *}\right) \Theta\left(\omega, T_{i}\right),
$$

where $\mathbf{P}_{(i)}^{(1,2)}$ is the dipole-quadrupole polarizability, given that

$$
P_{(i) ; \alpha, \beta}^{(1,2)}=\gamma_{(i)}(\omega) \square_{\alpha_{1}, \beta_{1}, \beta_{2}}^{(1)},
$$

with $\gamma_{(i)}(\omega)=\gamma_{(i)}(\omega)+i \gamma_{(i)}^{\prime \prime}(\omega)$ and

$$
\square_{\alpha_{1}, \beta_{1}, \beta_{2}}^{(1)}=\varepsilon_{\alpha_{1}, \beta_{1}, \beta_{2}},
$$

which is an isotropic skew-symmetric tensor. From Eqs. (23)-(25), it follows

$$
\left\langle M_{(i) ; \alpha}^{(1) *} M_{(i) ; \beta}^{(2)}\right\rangle=\frac{2 \varepsilon_{0}}{\pi \omega c_{2}} \gamma_{(i)}^{\|}(\omega) \Theta\left(\omega, T_{i}\right) \varepsilon_{\alpha_{1}, \beta_{1}, \beta_{2}} .
$$

It must be emphasized that the FDT [Eq. (15)] applies whenever the charge distribution of each particle in the presence of mutual interactions has reached equilibrium with the heat bath. When this is not the case, in the nonaged regime, ${ }^{11}$ one can still use a similar expression of the FDT in terms of an effective temperature. This can be done through a generalized Langevin equation, ${ }^{9}$ which takes into account the heat exchange between the NPi and its thermal bath. Relating the momentum variance of the NPi with its temperature by the equipartition theorem, one can obtain the effective temperature $\left(T_{e f f(i)}^{(l, k)}\right)$ through the response of the system due to fluctuations of the multipolar moments. ${ }^{16}$ Thus,

$$
T_{e f f(i)}^{(l, k)}=T_{0}+\left(T_{0}-T_{b}\right)\left[\left\langle\mathbf{M}_{(i)}^{(l) *} \odot \mathbf{M}_{(i)}^{(k)}\right\rangle^{2}-1\right],
$$

where $T_{0}$ stands for the initial temperature of the NPi and $T_{b}$ is the bath temperature. This expression shows that when the multipole moments of the particles are uncorrelated, i.e., when both particles equilibrate independently at two different temperatures, the effective temperature coincides with that of the bath. This is the situation addressed in this paper.

The effective temperature, defined as that for which the system would equilibrate, is a parameter measuring the distance to the stationary state in which both particles reach two different temperatures. It can also be calculated by using a relaxation model. ${ }^{17,18}$

\section{THERMAL CONDUCTANCE}

In this section, we will calculate the thermal conductance between the two NPs in the presence of quadrupolar contributions. To this end, we start by writing Eq. (14) as follows:

$$
\begin{aligned}
Q_{i \rightarrow j}= & \frac{-i \omega \varepsilon_{0}}{4} \sum_{l, k=0}^{\infty} c_{l} c_{k} \sum_{\{\beta\},\{\alpha\}} \sum_{(i) ; \beta}\left\langle M_{(i) ; \alpha}^{(l) *} M^{(k)}\right\rangle \\
& \times\left(\mathbf{S}_{(j) ; \beta, \alpha}^{(l, k)}-\mathbf{S}_{(j) ; \alpha, \beta}^{(k, l) *}\right),
\end{aligned}
$$

where from Eqs. (7), (9), and (13)

$$
\mathbf{S}_{(j) ; \beta, \alpha}^{(l, k)}=\frac{1}{\left(4 \pi \varepsilon_{0}\right)^{2}} \sum_{n, m=0}^{\infty} d^{-(n+m+l+k+2)} \times A_{(j)}^{(m, n)}(\omega) B_{\beta, \alpha}^{(l, r)}(\hat{\mathbf{d}}, \omega)
$$

with

$$
A_{(j)}^{(m, n)}(\omega) B_{\beta, \alpha}^{(l, k)}(\hat{\mathbf{d}}, \omega)=\sum_{\{\gamma\}} \sum_{\{\nu\}} Y_{\beta, \gamma}^{(l, n)}(\hat{\mathbf{d}}) P_{(j) ; \gamma, \nu}^{(n, m)}(\omega) \times Y_{\nu, \alpha}^{(m, k)}(\hat{\mathbf{d}}) .
$$

Hence, by substituting Eq. (30) into Eq. (29) and the resulting equation into Eq. (28), we obtain 


$$
Q_{i \rightarrow j}=\frac{1}{(4 \pi)^{3}} \sum_{l, k=0}^{\infty} c_{l} c_{k} \sum_{n, m=0}^{\infty} d^{-(n+m+l+k+2)} C_{(i \rightarrow j)}^{n, m, l, k}
$$

where

$$
C_{(i \rightarrow j)}^{n, m, l, k}=\frac{-i \omega \pi}{\varepsilon_{0}}\left\{A_{(j)}^{(m, n)}(\omega) \sum_{\{\beta\}} \sum_{\{\alpha\}} B_{\beta, \alpha}^{(l, k)}\left\langle M_{(i) ; \beta}^{(l) *} M_{(i) ; \alpha}^{(k)}\right\rangle-\text { c.c. }\right\} .
$$

Therefore, from Eq. (31), we obtain the net heat flux between both NPs,

$$
\begin{aligned}
Q_{12}= & Q_{1 \rightarrow 2}-Q_{2 \rightarrow 1}=\frac{1}{(4 \pi)^{3}} \sum_{l, k=0}^{\infty} c_{l} c_{k} \sum_{n, m=0}^{\infty} d^{-(n+m+l+k+2)} \\
& \times\left(C_{(1 \rightarrow 2)}^{n, m, l, k}-C_{(2 \rightarrow 1)}^{n, m, l, k}\right) .
\end{aligned}
$$

In view of Eq. (25) and the symmetric character of the spherical surface tensors given in Eq. (A3), one can prove from Eq. (30) that

$$
A_{(j)}^{(2,1)}(\omega) B_{\beta, \alpha}^{(l, k)}=A_{(j)}^{(1,2)}(\omega) B_{\beta, \alpha}^{(l, k)}=0 .
$$

Moreover, it can be shown that when $m+n=2 p+1(p>n)$, $\mathbf{P}_{(j)}^{(n, m)}$ is proportional to an isotropic skew-symmetric tensor ${ }^{(p)}$ of order $2 p+1$ which satisfies ${ }^{15}$

$$
\square_{\mu, \lambda, \mu}^{(p)}=-\square_{\mu, \lambda, \mu}^{(p)} .
$$

Therefore, by symmetry reasons, only coefficients $C_{(i \rightarrow j)}^{n, m, l, k}$ for which $n+m=2 q$ and $l+k=2 s$, with $q$ and $s$ as two positive integers, contribute to the heat flux. Hence, up to quadrupolar order, we can write from Eq. (31)

$$
\begin{aligned}
Q_{i \rightarrow j}= & \frac{1}{(4 \pi)^{3}}\left\{C_{(i \rightarrow j)}^{1,1,1,1} d^{-6}+\left(C_{(i \rightarrow j)}^{1,1,2,2}+\cdots+9 C_{(i \rightarrow j)}^{2,2,1,1}\right) d^{-8}\right. \\
& \left.+C_{(i \rightarrow j)}^{2,2,2,2} d^{-10}+\cdots\right\} .
\end{aligned}
$$

Therefore, from Eqs. (32), (36), and (A8)-(A11), we arrive at

$$
\begin{aligned}
Q_{i \rightarrow j}= & \frac{3}{8 \pi^{3}}\left\{\alpha_{(i)}^{\prime \prime} \alpha_{(j)}^{\prime \prime} d^{-6}+15\left(\alpha_{(j)}^{\prime \prime} \beta_{(i)}^{\prime \prime}+3 \alpha_{(i)}^{\prime \prime} \beta_{(j)}^{\prime \prime}\right) d^{-8}\right. \\
& \left.+140 \beta_{(i)}^{\prime \prime}(\omega) \beta_{(j)}^{\prime \prime}(\omega) d^{-10}+\cdots\right\} \Theta\left(\omega, T_{i}\right),
\end{aligned}
$$

and consequently,

$$
\begin{aligned}
Q_{12}(\omega)= & Q_{1 \rightarrow 2}-Q_{2 \rightarrow 1}=\frac{3}{8 \pi^{3}}\left\{\alpha_{(1)}^{\prime \prime} \alpha_{(2)}^{\prime \prime} d^{-6}\right. \\
& \left.+140 \beta_{(1)}^{\prime \prime}(\omega) \beta_{(2)}^{\prime \prime}(\omega) d^{-10}\right\} \Delta \Theta \\
& +\frac{45}{8 \pi^{3}}\left\{\left(\alpha_{(2)}^{\prime \prime} \beta_{(1)}^{\prime \prime}+3 \alpha_{(1)}^{\prime \prime} \beta_{(2)}^{\prime \prime}\right) \Theta\left(\omega, T_{1}\right)\right. \\
& \left.-\left(\alpha_{(1)}^{\prime \prime} \beta_{(2)}^{\prime \prime}+3 \alpha_{(2)}^{\prime \prime} \beta_{(1)}^{\prime \prime}\right) \Theta\left(\omega, T_{2}\right)\right\} d^{-8},
\end{aligned}
$$

where $\Delta \Theta \equiv\left\{\Theta\left(\omega, T_{1}\right)-\Theta\left(\omega, T_{2}\right)\right\}$.

When NPs are at the same temperature $T$, Eq. (38) reduces to

$$
Q_{12}(\omega)=\frac{45}{4 \pi^{3}}\left(\alpha_{(1)}^{\prime \prime} \beta_{(2)}^{\prime \prime}-\alpha_{(2)}^{\prime \prime} \beta_{(1)}^{\prime \prime}\right) d^{-8} \Theta(\omega, T),
$$

since the system is in thermal equilibrium,

$$
\int_{0}^{\infty} Q_{12}(\omega) d \omega=0
$$

In the general case, i.e., out of equilibrium, we can linearize Eq. (38) with respect to the temperature diference $\Delta T$ $=T_{1}-T_{2}$ in order to obtain the conductance given through $G_{12}\left(T_{0}\right)=\partial Q_{12} /\left.\partial \Delta T\right|_{T_{1}=T_{2}=T_{0}}$. We obtain

$$
\begin{aligned}
G_{12}\left(T_{0}\right)= & \frac{3}{8 \pi^{3}} \int_{0}^{\infty} \Theta^{\prime}\left(\omega, T_{0}\right)\left\{\alpha_{(1)}^{\prime \prime} \alpha_{(2)}^{\prime \prime} d^{-6}\right. \\
& +60\left(\alpha_{(1)}^{\prime \prime} \beta_{(2)}^{\prime \prime}+\alpha_{(2)}^{\prime \prime} \beta_{(1)}^{\prime \prime}\right) d^{-8} \\
& \left.+5 \beta_{(1)}^{\prime \prime}(\omega) \beta_{(2)}^{\prime \prime}(\omega) d^{-10}\right\} d \omega
\end{aligned}
$$

where $T_{0}=\left(T_{1}+T_{2}\right) / 2$ is the average temperature, which corresponds to the final equilibrium temperature that two bodies would reach when brought into contact and a heat flow established between them. ${ }^{19}$

In the expression we have obtained for the conductance, we can identify the following contributions.

(i) For dipolar,

$$
G_{12}^{\operatorname{dip}}\left(T_{0}\right)=\frac{3}{8 \pi^{3}}\left(\int_{0}^{\infty} \Theta^{\prime}\left(\omega, T_{0}\right) \alpha_{(1)}^{\prime \prime} \alpha_{(2)}^{\prime \prime} d \omega\right) d^{-6},
$$

which coincides with the expression obtained in Ref. 7.

(ii) For quadrupolar,

$$
\begin{aligned}
G_{12}^{q d}\left(T_{0}\right)= & \frac{1}{2 \pi^{3}} \int_{0}^{\infty} \Theta^{\prime}\left(\omega, T_{0}\right)\left\{45\left(\alpha_{(1)}^{\prime \prime} \beta_{(2)}^{\prime \prime}+\alpha_{(2)}^{\prime \prime} \beta_{(1)}^{\prime \prime}\right) d^{-8}\right. \\
& \left.+\frac{15}{4} \beta_{(1)}^{\prime \prime}(\omega) \beta_{(2)}^{\prime \prime}(\omega) d^{-10}\right\} d \omega .
\end{aligned}
$$

In order to verify our results, in Fig. 2, we reexhibit a graph obtained by Domingues et al., ${ }^{7}$ extending the logarithmic scale for conductance in the more usual form. This graph displays the thermal conductance as a function of distance between the NPs, both with radius $R$, in three significant situations: in mechanical contact $(d=2 R)$, in the intermediate region shortly before contact $(2 R<d<4 R)$, and in the most distant region $(d \geqslant 4 R)$ where the near-field interaction is still valid. In this situation, the results corresponding to the gray dotted lines show the behavior $d^{-6}$ which was obtained in Ref. 7. Our results are in broad agreement for this region where the dipolar domain is present. When the particles are close togheter, their charge distributions become very disorderly and higher orders than dipolar interactions come into play in the calculation of the thermal conductance. In this case, as predicted by Domingues et al., ${ }^{7}$ the thermal conductance is about 4 orders of magnitude larger than that of the dipole model given in Eq. (42). In more extreme conditions when the particles come into contact to each other, the same authors also predicted that the conductance would be 2-3 orders of magnitude lower than the conductance just before 


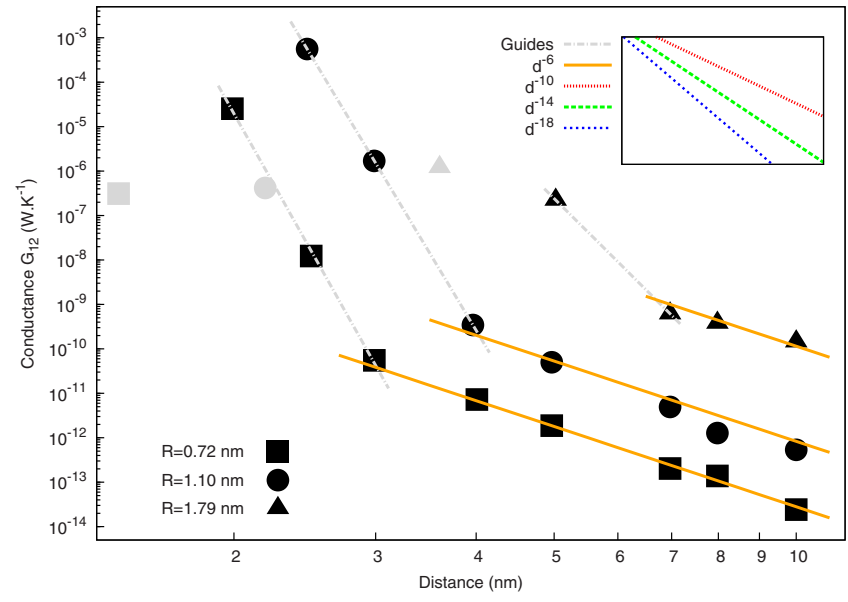

FIG. 2. (Color online) Thermal conductance $G_{12}$ vs distance $d$ reproducing the molecular dynamics data obtained by Domingues et al. (Ref. 7) The gray points represent the conductance when the particles with effective radii of $0.72,1.10$, and $1.79 \mathrm{~nm}$ are in contact. The dotted lines show the analytical result obtained by these authors. The values of the conductance at short distances have been given through the gray dash-dotted lines. The inset shows different guide behaviors as a function of the distance having different values of the exponents.

contact. At contact, in addition to the Coulomb interactions, the interchange of phonons also contributes to the energy transfer, compensating the higher order terms of the multipolar expansion. Moreover, as we have discussed after Eq. (34), due to the tensorial character of the polarizabilities, only even multipoles contribute to the multipolar expansion. These numerical predictions are contained the result we give in Eq. (41), where one can see that the dominant contribution $\left(d^{-10}\right)$ is 4 orders of magnitude lower than the dipolar case $\left(d^{-6}\right)$, while an intermediate case would give a value $d^{-8}$. It must be stressed that we have obtained the conductance up to quadrupolar order; nonetheless, through our formalism, it is possible to obtain the conductance for any order of multipolar interaction.

\section{CONCLUSIONS}

In this paper, we have presented a theory to explain the exchange of energy between two NPs at different temperatures. Our theory provides a general formalism based on the multipolar expansion of the electrostatic field in order to study heat transfer between two NPs for arbitrary small distances provided that the FDT is satisfied. However, out of the FDT regime and when the system possesses fast and slow degrees of freedom, it is possible to formulate a FDT in terms of a nonstationary effective temperature which depends on the slow degrees of freedom. ${ }^{17,18}$

We have found that our analysis of the heat interchanged between two NPs separated by a few submicrons agrees with the explainations of the rapid growth of the conductance observed in the simulation ${ }^{7}$ even when the NPs are in contact. Hence, we are able to provide a comprehensive explanation of the numerical results reported in Ref. 7.
The formalism presented could also be applied to other situations such as the radiative heat transfer between a small dielectric particle and a surface ${ }^{20}$ and the study of the optical forces due the radiation of a thermal source, ${ }^{21}$ enabling us to go beyond the dipolar approximation.

\section{ACKNOWLEDGMENTS}

One of the authors (J.M.R.) wants to thank J. J. Greffet for interesting discussions. This work was supported by the DGiCYT of Spanish Government under Grant No. FIS200501299 and by Brazilian Research Foundations: CAPES and CNPq.

\section{APPENDIX A}

In this Appendix, we present some of the properties of the spherical surface tensors $Y_{\alpha}^{(n)}(\hat{\mathbf{r}})$ related to the $n$-rank Cartesian tensor,

$$
X_{\alpha}^{(n)}(\mathbf{r})=(-1)^{n} \frac{\partial^{n}}{\partial a_{\alpha_{1}} \cdots \partial a_{\alpha_{n}}} \frac{1}{r},
$$

introduced in Eq. (1). The tensor $X_{\alpha}^{(n)}(\mathbf{r})$ are given in terms of the unit vector $\hat{\mathbf{r}}$ related to $\mathbf{r}$ as ${ }^{15}$

$$
X_{\alpha}^{(n)}(\mathbf{r})=r^{-(n+1)} Y_{\alpha}^{(n)}(\hat{\mathbf{r}}) .
$$

This spherical surface tensors can be expressed as

$$
Y_{\alpha}^{(n)}(\hat{\mathbf{r}})=(2 n-1) ! ! \overline{\hat{r}_{\alpha_{1}} \cdots \hat{r}_{\alpha_{n}}},
$$

where $\overline{\hat{r}_{\alpha_{1}} \cdots \hat{r}_{\alpha_{n}}}$ are the symmetric irreducible tensor constructed with the components of $\hat{\mathbf{r}}$. The first three symmetric irreducible tensors are

$$
\begin{gathered}
\overline{\hat{r}_{\alpha}}=r^{-1} r_{\alpha}, \\
\overline{\hat{r}_{\alpha_{1}} \hat{r}_{\alpha_{2}}}=r^{-2}\left(r_{\alpha_{1}} r_{\alpha_{2}}-\frac{1}{3} \delta_{\alpha_{1} \alpha_{2}}\right), \\
\overline{\hat{r}_{\alpha_{1}} \hat{r}_{\alpha_{2}} \hat{r}_{\alpha_{3}}}=r^{-3}\left[r_{\alpha_{1}} r_{\alpha_{2}} r_{\alpha_{3}}-\frac{1}{5} r^{2}\left(\delta_{\alpha_{1} \alpha_{2}{ }_{\alpha_{3}}} r_{\alpha_{1} \alpha_{3}} r_{\alpha_{2}}\right.\right. \\
\left.\left.+\delta_{\alpha_{2} \alpha_{3}} r_{\alpha_{1}}\right)\right] .
\end{gathered}
$$

The spherical surface tensors satisfy the following property: ${ }^{15}$

$$
\sum_{\{\alpha\}} Y_{\alpha}^{(n)}(\hat{\mathbf{r}}) Y_{\alpha}^{(n)}(\hat{\mathbf{r}})=(2 n-1) ! ! n ! .
$$

By using Eq. (30), we can obtain the coefficients defined in Eq. (32). From Eqs. (16)-(22), (32), and (A7), one has

$$
\begin{aligned}
C_{(i \rightarrow j)}^{1,1,1,1} & =\frac{-i \omega \pi}{\varepsilon_{0}}\left\{A_{(j)}^{(1,1)}(\omega) \sum_{\{\beta\}} \sum_{\{\alpha\}} B_{\beta, \alpha}^{(1,1)}\left\langle M_{(i) ; \beta}^{(1) *} M_{(i) ; \alpha}^{(1)}\right\rangle-\text { c.c. }\right\} \\
& =4 \alpha_{(i)}^{\prime \prime} \alpha_{(j)}^{\prime \prime} \sum_{\{\beta\}} \sum_{\{\nu\}} Y_{\beta, \nu}^{(1,1)} Y_{\beta, \nu}^{(1,1)} \Theta\left(\omega, T_{i}\right) \\
& =24 \alpha_{(i)}^{\prime \prime} \alpha_{(j)}^{\prime \prime} \Theta\left(\omega, T_{i}\right) .
\end{aligned}
$$

and 


$$
\begin{aligned}
C_{(i \rightarrow j)}^{2,2,2,2} & =\frac{-i \omega \pi}{\varepsilon_{0}}\left\{A_{(j)}^{(2,2)} \sum_{\{\beta\}} \sum_{\{\alpha\}} B_{\beta, \alpha}^{(2,2)}\left\langle M_{(i) ; \beta}^{(2)} M_{(i) ; \alpha}^{(2) *}\right\rangle-\text { c.c. }\right\} \\
& =4 \beta_{(i)}^{\prime \prime}(\omega) \beta_{(j)}^{\prime \prime}(\omega) \sum_{\{\beta\}} \sum_{\{\nu\}} Y_{\beta, \nu}^{(2,2)} Y_{\beta, \nu}^{(2,2)} \Theta\left(\omega, T_{i}\right) \\
& =4(7 ! !)(4 !) \beta_{(i)}^{\prime \prime}(\omega) \beta_{(j)}^{\prime \prime}(\omega) \Theta\left(\omega, T_{i}\right) .
\end{aligned}
$$

The remaining coefficients are obtained in similar way,

$$
\begin{aligned}
C_{(i \rightarrow j)}^{1,1,2,2} & =\frac{-i \omega \pi}{\varepsilon_{0}}\left\{A_{(j)}^{(1,1)}(\omega) \sum_{\{\beta\},\{\alpha\}} \sum_{\beta, \alpha} B_{\beta, 2)}^{(2,2)}\left\langle M_{(i) ; \beta}^{(2) *} M_{(i) ; \alpha}^{(2)}\right\rangle-\text { c.c. }\right\} \\
& =4 \alpha_{(j)}^{\prime \prime}(\omega) \beta_{(i)}^{\prime \prime}(\omega) \sum_{\{\beta\},\{\alpha\}} Y_{\beta, \nu}^{(2,1)} Y_{\nu, \beta}^{(1,2)} \\
& =360 \alpha_{(j)}^{\prime \prime}(\omega) \beta_{(i)}^{\prime \prime}(\omega) \Theta\left(\omega, T_{i}\right)
\end{aligned}
$$

and

$$
C_{(i \rightarrow j)}^{2,2,1,1}=360 \alpha_{(i)}^{\prime \prime}(\omega) \beta_{(j)}^{\prime \prime}(\omega) \Theta\left(\omega, T_{j}\right) .
$$

\section{APPENDIX B}

This Appendix is devoted to the derivation of the expression of the energy dissipated corresponding to Eq. (11). In the adiabatic case, for a perturbation of the form,

$$
\hat{H}=-c_{j} \hat{x}_{j} f_{j}(t),
$$

where $\hat{x}_{j}$ is a generalized displacement and $f_{j}(t)$ is a generalized force. The change in the energy of the system is equal to the mean value of the partial derivative of the Hamiltonian with respect to time. Since only the perturbation $\hat{H}$ in the Hamiltonian depends explicitly on time and $\hat{x}_{j}$ is a dynamical observable of the system which is independent of time, we have

$$
d E / d t=-c_{j} x_{j} d f_{j} / d t .
$$

In the framework of linear response theory, one assumes that

$$
c_{j} x_{j}(t)=\int_{0}^{\infty} \alpha_{j k}(\tau) f_{k}(t-\tau) d \tau,
$$

which is a relation similar to Eq. (10). After introducing the Fourier transforms and combining Eqs. (B3) and (B2), we can write

$$
\begin{aligned}
\frac{d E}{d t}= & \frac{1}{(2 \pi)^{2}} \int d \omega \int d \omega^{\prime} \exp \left[-i\left(\omega+\omega^{\prime}\right) t\right] \\
& \times\left(i \omega^{\prime}\right) f_{j}\left(\omega^{\prime}\right) \alpha_{j k}(\omega) f_{k}(\omega) .
\end{aligned}
$$

Here, if the perturbation $\mathbf{f}$ acts over a finite time, the total energy dissipated is

$$
\begin{aligned}
\int_{-\infty}^{\infty} d t \frac{d E}{d t}= & \frac{2}{2 \pi} \int d \omega \int d \omega^{\prime} \delta\left(\omega+\omega^{\prime}\right) \\
& \times\left(i \omega^{\prime}\right) f_{j}\left(\omega^{\prime}\right) \alpha_{j k}(\omega) f_{k}(\omega) \\
= & \frac{1}{2 \pi} \int d \omega(-i \omega) f_{j}(-\omega) \alpha_{j k}(\omega) f_{k}(\omega) .
\end{aligned}
$$

Since the total heat must be a real quantity,

$$
\begin{aligned}
& \frac{1}{2 \pi} \int d \omega(-i \omega) f_{j}(-\omega) \alpha_{j k}(\omega) f_{k}(\omega) \\
& \quad=-\frac{i}{4 \pi} \int d \omega\left(f_{j}^{*} \alpha_{j k} f_{k}-f_{j} \alpha_{j k}^{*} f_{k}^{*}\right) \omega .
\end{aligned}
$$

Therefore, the heat at the frequency $\omega$ is given through

$$
\hat{Q}(\omega)=-\frac{i \omega}{4 \pi}\left(f_{j}^{*} \alpha_{j k} f_{k}-f_{k} \alpha_{k j}^{*} f_{j}^{*}\right),
$$

which after performing the thermal average leads to the equation equivalent to Eq. (11),

$$
Q(\omega)=-\frac{i \omega}{4 \pi}\left(\left\langle f_{j}^{*} \alpha_{j k} f_{k}\right\rangle-\left\langle f_{k} \alpha_{k j}^{*} f_{j}^{*}\right\rangle\right)
$$

\footnotetext{
*agustiperezmadrid@ub.edu

†mrubi@ub.edu

Huciano@fis.unb.br

${ }^{1}$ Microscale and Nanoscale Heat Transfer, edited by S. Volz, Topics in Applied Physics Vol. 107 (Springer, Berlin, 2007).

${ }^{2}$ K. Joulain, J. P. Mulet, F. Marquier, R. Carminati, and J. J. Greffet, Surf. Sci. Rep. 57, 59 (2005).

${ }^{3}$ J. M. G. Vilar and J. M. Rubí, Proc. Natl. Acad. Sci. U.S.A. 98, 11081 (2001)

${ }^{4}$ D. Reguera, J. M. Rubí, and J. M. G. Vilar, J. Phys. Chem. B 109, 21502 (2005).

${ }^{5}$ T. L. Hill, Thermodynamics of Small Systems (Dover, New York, 2002).

${ }^{6}$ A. I. Volokitin and B. N. J. Persson, Rev. Mod. Phys. 79, 1291 (2007).

${ }^{7}$ G. Domingues, S. Volz, K. Joulain, and J. J. Greffet, Phys. Rev. Lett. 94, 085901 (2005).
}

${ }^{8}$ H. B. Callen and T. A. Welton, Phys. Rev. 83, 34 (1951).

${ }^{9}$ R. Kubo, M. Toda, and N. Hashitsume, Statistical Physics II: Nonequilibrium Statistical Mechanics, Springer Series in SolidState Science Vol. 31 (Springer, Berlin, 1985).

${ }^{10}$ L. D. Landau and E. M. Lifshitz, in Statistical Physics, 3rd ed. (Pergamon, Oxford, 1980), Vol. 5, Pt. 1, p. 389.

${ }^{11}$ S. R. de Groot and P. Mazur, Non-Equilibrium Thermodynamics (Dover, New York, 1984), p. 156.

${ }^{12}$ T. Forster, Ann. Phys. 2, 55 (1948).

${ }^{13}$ R. Carminati, J. J. Greffet, C. Henkel, and J. M. Vigoureux, Opt. Commun. 261, 368 (2006).

${ }^{14}$ A. J. Stone, in The Theory of Intermolecular Forces (Clarendon, Oxford, 1996).

${ }^{15} \mathrm{~S}$. Hess and W. Köhler, Formeln zur Tensor-Rechnung (Palm \& Enke, Erlangen, 1980).

${ }^{16}$ L. C. Lapas, I. V. L. Costa, M. H. Vainstein, and F. A. Oliveira, 
Europhys. Lett. 77, 37004 (2007).

${ }^{17}$ A. Pérez-Madrid, D. Reguera, and J. M. Rubí, Physica A 329, 357 (2003).

${ }^{18}$ A. Pérez-Madrid, J. Chem. Phys. 122, 214914 (2005).

${ }^{19}$ C. J. Adkins, Equilibrium Thermodynamics, 3rd ed. (Cambridge
University Press, Cambridge, 1994).

${ }^{20}$ J. P. Mulet, K. Joulain, R. Carminati, and J. J. Greffet, Appl. Phys. Lett. 78, 2931 (2001).

${ }^{21}$ C. Henkel, K. Joulain, J. P. Mulet, and J. J. Greffet, J. Opt. A, Pure Appl. Opt. 4, S109 (2002). 